\title{
A New Dimension Analysis on Blocking Behavior in Banyan-Based Optical Switching Networks
}

\author{
Chen $\mathrm{YU}^{\dagger \mathrm{a})}$, Nonmember, Yasushi INOGUCHI ${ }^{\dagger}$, and Susumu HORIGUCHI ${ }^{\dagger \dagger}$, Members
}

\begin{abstract}
SUMMARY Vertically stacked optical banyan (VSOB) is an attractive architecture for constructing banyan-based optical switches. Blocking behaviors analysis is an effective approach to studying network performance and finding a graceful compromise among hardware costs, blocking probability and crosstalk tolerance; however, little has been done on analyzing the blocking behavior of VSOB networks under crosstalk constraint which adds a new dimension to the switching performance. In this paper, we study the overall blocking behavior of a VSOB network under various degree of crosstalk, where an upper bound on the blocking probability of the network is developed. The upper bound depicts accurately the overall blocking behavior of a VSOB network as verified by extensive simulation results and it agrees with the strictly nonblocking condition of the network. The derived upper bound is significant because it reveals the inherent relationship between blocking probability and network hardware cost, by which a desirable tradeoff can be made between them under various degree of crosstalk constraint. Also, the upper bound shows how crosstalk adds a new dimension to the theory of switching systems.

key words: optical switching networks, banyan networks, blocking probability, vertical stacking optical banyan, degree of crosstalk
\end{abstract}

\section{Introduction}

Optical mesh networks are considered increasingly capacityefficient and survivable for serving as network backbone for the next generation Internet. All-Optical switches serve as key network elements in such an environment by automatically steering network traffic at an ultra-high speed. The basic $2 \times 2$ switching element (SE) in optical switching networks is usually a directional coupler (DC) [1], [2]. A DC can simultaneously switch optical flows with multiple wavelengths, and is one of the best candidates for serving as a SE for the future optical cross-connects (OXCs) to support Optical Burst Switching (OBS) and Optical Packet Switching (OPS).

Crosstalk is an intrinsic shortcoming of the DC. It is the effect of the undesirable coupling between the signals carried in the two waveguides of the coupler [1], [3]. When two optical signals meet at a DC, a small portion of the signal power will be directed to the unintended output channel. Crosstalk suppression becomes particularly important in networks, where a signal propagates through many nodes and accumulates crosstalk from different elements at

Manuscript received January 7, 2008.

Manuscript revised March 5, 2008.

${ }^{\dagger}$ The authors are with Japan Advanced Institute of Science and Technology, Nomi-shi, 923-1211 Japan.

${ }^{\dagger \dagger}$ The author is with Tohoku University, Sendai-shi, 980-8579 Japan.

a) E-mail: yuchen0604@hotmail.com

DOI: 10.1093/ietisy/e91-d.7.1991 each node from the system view. In order to obtain an approximate idea of the crosstalk requirements, suppose that a signal accumulates crosstalk from $N$ sources, each with crosstalk level $\varepsilon$. This neglects the fact that some interfering channels may have higher powers than the desired channel. Networks are very likely to contain amplifiers and to be limited by signal-spontaneous beat noise. For example, if we have 10 interfering equal-power crosstalk elements, each producing intrachannel crosstalk, then we must have a crosstalk suppression of below $35 \mathrm{~dB}$ in each element, in order to have an overall penalty of less than $1 \mathrm{~dB}$ [2]. Thus, crosstalk reduction is an important issue in designing the systems that are based on DC's. The crosstalk issue can be tackled at either the device level or the system level. The two methods complement each other. The focus of this paper is on the system-level approach. As shown by the analytical model, crosstalk adds a new dimension to the theory of building a nonblocking or a negligible blocking VSOB network.

Banyan networks [4]-[7] are a class of attractive switching structures for constructing DC-based optical switches because they have a smaller and exact same number of SEs along any path between an input-output pair; therefore, an absolutely loss uniformity and smaller attenuation of optical signals are guaranteed. However, banyan networks are blocking networks, and a general approach to building banyan-based nonblocking optical switching networks is to vertically stack the multiple copies of banyan [8], [9]. We use VSOB to denote the optical switching networks built on the vertical stacking of optical banyan networks. In this paper, we focus on the VSOB networks that are under various degree of crosstalk constraint $c$, where $0<c \leq \log N / 2^{*}$ according to the real implementation.

Numerous results are available on the study of VSOB networks, such as [10]-[12], and their main focus has been on determining the minimum number of stacked copies (planes) required for a nonblocking VSOB network. These results indicate that the VSOB structure, although is attractive, usually requires either a high hardware cost or and a large network depth to achieve nonblockingness. Blocking behavior analysis of a network is an effective approach to studying network performance and finding desirable tradeoff between hardware costs and blocking probability. Some analytical models have been developed to understand the blocking behaviors of vertically stacked opti-

\footnotetext{
${ }^{*}$ In this paper log means the logarithm to the base 2 .
} 
cal banyan networks under crosstalk-free constraint that do not meet the nonblocking condition (i.e., with fewer stacked copies than required by the nonblocking condition) [10][12]. To our best knowledge, however, no study has been reported for modeling and evaluating the performance behavior of VSOB networks under various degree of crosstalk constraint. Thus, this paper is committed to analyzing blocking probability of a VSOB network by deriving its upper bound with respect to the number of planes in the network. The model can guide network designers to evaluate the overall blocking behavior of a VSOB network adopting different routing strategies, in which a graceful compromise can be initiated between the hardware cost and the blocking probability. The model can also show clearly how the crosstalk add a new dimension in building VSOB networks and guide us in making the design tradeoff among the degree of crosstalk, the hardware cost and the blocking probability.

\section{Preliminaries}

A typical $N \times N$ banyan network has $\log N$ stages and one unique path between any input-output pair. One basic technique for creating multiple paths between an input-output pair is the vertical stacking, where multiple banyan networks are vertically stacked, as illustrated in Fig. 1.

Due to their symmetry structures, all paths in a banyan networks have the same property in terms of blocking. We define the blocking probability to be the probability that a feasible connection request is blocked, where a feasible connection request is a connection request between an idle input port and an idle output port of the network. Without loss of generality, we chose the path between the first input and the first output (which is termed as the tagged path in the following context) for blocking analysis. All the SEs on the tagged path are called tagged SEs. The stages of SEs are numbered from left (stage 1) to right (stage $\log N$ ). For the tagged path, an input intersecting set $I_{i}$ associated with stage $i$ is defined as the set of all inputs that intersect a tagged SE at stage $i$. Likewise, an output intersecting set $O_{i}$ associated with stage $i$ is the set of all outputs that intersect a tagged SE at stage $\log N-i+1$.

When two light signals go through an SE simultane-

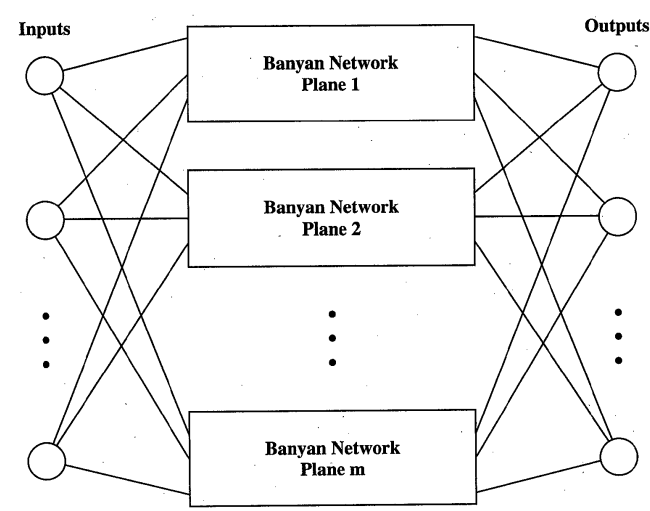

Fig. 1 Illustration of the vertical stacked optical banyan network. ously, crosstalk is generated at the SE. Such SE is referred to as a crosstalk SE (CSE). The degree of crosstalk of the switching system is defined as the number of CSE's allowed along a path. The crosstalk generated at each CSE can be found in the data sheet from the manufacturer.

A restricted SE (RSE) is a $2 \times 2$ SE which carries only one light signal at a time. Although crosstalk at an RSE is very small, it may not be entirely zero. For example, when a light signal passes through an RSE, a small portion of the signal will leave at the other unintended output channel. This stray signal can arrive at the input of the next stage SE and generate some crosstalk. Since crosstalk generated by the stray signal is much smaller than the regular crosstalk, we will ignore it in our analysis [10].

Following the typical assumption as in [12], [13] on probabilistic analysis of multistage interconnection networks, we neglect the correlation among signals arriving at input and outputs ports, and consider that the statuses (busy or idle) of individual input and output ports in the network are independent. This assumption is justified by the fact that the correlation among signals at inputs and outputs, though exists for fixed communication patterns, and becomes negligible for arbitrary communication patterns in large size networks, which is the trend of future optical switching networks that can switch huge data at high speeds.

\section{Upper Bound on Blocking Probability}

For simplicity, we use $\operatorname{VSOB}(N, m, c)$ to denote an $N \times N$ VSOB network that has $m$ stacked copies (planes) of an $N \times$ $N$ banyan network allows $c$ CSEs along the path, where $0<$ $c \leq \log N / 2$.

We take $\operatorname{NBP}(N, c)$ to denote the number of blocked planes in a $\operatorname{VSOB}(N, m, c)$ network under a "conservative" routing control strategy, in which all these connections that block a tagged path should fall within distinct planes to guarantee the nonblocking property. Here, we define plane as a blocked plane if all its tagged paths are blocked.

In this section, we first introduce the deterministic condition for the strictly nonblocking $\operatorname{VSOB}(N, m, c)$ that is obtained by finding the maximum value of $\operatorname{NBP}(N, c)$, then we develop the upper bound on blocking probability of a $\operatorname{VSOB}(N, m, c)$ network for the cases of even and odd numbers of stages, respectively.

\subsection{Conditions for Strictly Nonblocking}

Let the maximum value of $N B P(N, c)$ be $\max \{N B P(N, c)\}$, then $\operatorname{aSOB}(N, m, c)$ is strictly nonblocking if $m \geq 1+$ $\max \{N B P(N, c)\}[10]$. Thus, we only need to evaluate $\max \{N B P(N, c)\}$ for determining the nonblocking condition. The exact maximum value of $\operatorname{NBP}(N, c)$ has been studied in [10]. Here, we study the maximum value of $N B P(N, c)$ from a different perspective, which can be used to prove Theorem 1.

Lemma 1: The maximum value of $\operatorname{NBP}(N, c)$ is $(3 / 2) \sqrt{N}+$ $\lfloor\sqrt{N} /(c+1)\rfloor$, if $0<c \leq(1 / 2) \log N$ when $c$ is even. When $c$ 


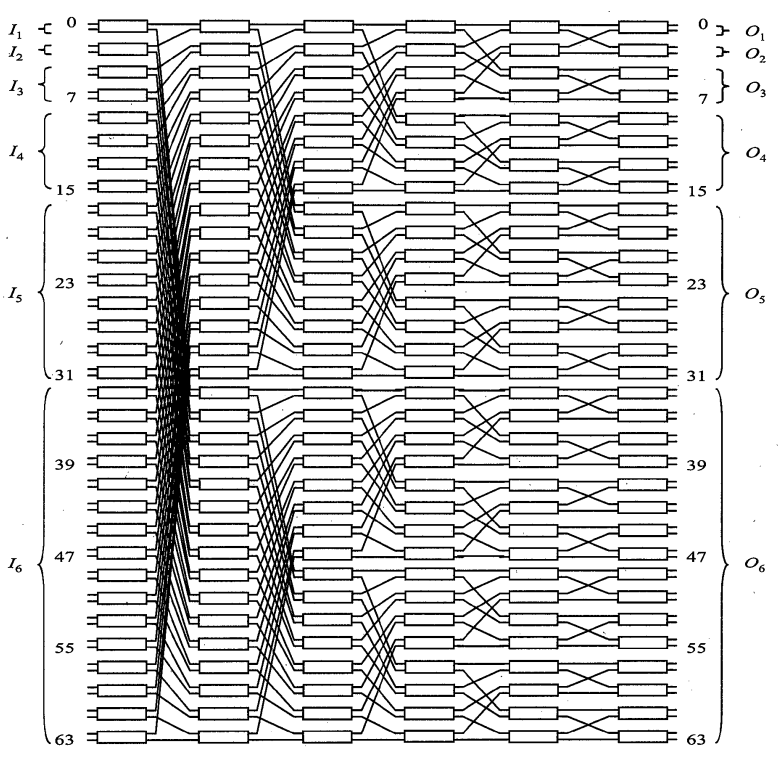

Fig. $264 \times 64$ banyan network.

is odd, the maximum value becomes $\sqrt{2 N}+\lfloor\sqrt{2 N} /(c+2)\rfloor$, if $0<c \leq(\log N-1) / 2$.

Proof: As shown in Fig. 2, for the case when $\log N$ is even, we further define input set $I_{\text {even }}$ and output set $O_{\text {even }}$ as:

$$
I_{\text {even }}=\bigcup_{i=1}^{\frac{1}{2} \log N} I_{i}, \quad O_{\text {even }}=\bigcup_{i=1}^{\frac{1}{2} \log N} O_{i}
$$

Where the input intersecting set $I_{i}$ associated with stage $i$ is defined as the set of all inputs that intersect a tagged SE at stage $i$ and the output intersecting set $O_{i}$ associated with stage $i$ is the set of all outputs that intersect a tagged SE at stage $\log N-i+1$.

Under the constraint of crosstalk $c$, the blocking behaviors on the tagged path consist of two parts: $L(N)$ regardless $c$ (which means the number of connection requests block the tagged path in terms of link-blocking) and $C(N, c)$ regard $c$ (which means the number of connection requests block the tagged path in terms of crosstalk-blocking). The worst-case scenario of conflicts on the tagged path is when all inputs in set $I_{i}$ are destined for the outputs in set $O_{(\log N-i+1)}$ and all outputs in set $O_{i}$ are originated from the inputs in set $I_{(\log N-i+1)}$. Thus, the maximum number of conflicts with the tagged path is determined by both the connections from set $I_{\text {even }}$ and the connections destined for set $O_{\text {even }}$ which cause $L(N)$ and $C(N, c)$ separately.

Here, we focus on the maximum value of $\operatorname{NBP}(N, c)$ when $c \geq 1$. Let $L(N)$ be the number of planes blocked by the connections from $I_{i}(1 \leq i \leq(1 / 2) \log N-1)$ and/or destined for $O_{i}(1 \leq i \leq(1 / 2) \log N-1)$. Then we have the $\max \{L(N)\}=\left|I_{1}\right|+\cdots+\left|I_{(1 / 2) \log N-1}\right|+\left|O_{1}\right|+\cdots+$ $\left|O_{(1 / 2) \log N-1}\right|+(1 / 2)\left(\left|I_{(1 / 2) \log N}\right|+\left|O_{(1 / 2) \log N}\right|\right)=(3 / 2) \sqrt{N}$, and $\max \{C(N, c)\}=\lfloor\sqrt{N} /(c+1)\rfloor[10]$.

The proof for the case when $\log N$ is odd is the same as for the case when $\log N$ is even, after taking out all connections passing through the middle stage on the tagged path and considering them separately from the remaining $\log N-1$ stages. The $\max \{L(N)\}=\left|I_{1}\right|+\cdots+\left|I_{(\log N \pm 1) / 2}\right|+$ $\left|O_{1}\right|+\cdots+\left|O_{(\log N \pm 1) / 2}\right|=\sqrt{2 N}$, and $\max \{C(N, c)\}=$ $\lfloor\sqrt{2 N} /(c+2)\rfloor[11]$. QED.

\subsection{Upper Bound on Blocking Probability When the Num- ber of Stages is Even}

The upper bound for $\operatorname{VSOB}(N, m, 0)$ network has been developed in [12]. In this paper, we focus on the $\operatorname{VSOB}(N, m, c)$ network when $c \geq 1$. We use $\operatorname{Pr}(A)$ to denote the probability that the event $A$ happens and use $\operatorname{Pr}^{+}(A)$ to denote the upper bound of $\operatorname{Pr}(A)$. Based on Lemma 1, the blocking probability $\operatorname{Pr}^{+}$(blocking) for a $\operatorname{VSOB}(N, m, c)$ network, where $\log N$ is even (please refer to Fig. 2), is given by:

$$
\begin{aligned}
& \operatorname{Pr}^{+} \text {(blocking) } \\
& =1-\sum_{d=0}^{\min \{(3 / 2) \sqrt{N}+\lfloor\sqrt{N} /(c+1)\rfloor, m-1\}} \operatorname{Pr}(N B P(N, c)=d)
\end{aligned}
$$

Since the "conservative" routing control strategy, in which each set of these connections that block a tagged path falls within a distinct plane, has been used in determining $\operatorname{NBP}(N, c)$, the blocking probability of a connection request under any routing control strategy is then upper-bounded by the blocking probability given in (1).

Equation (1) indicates clearly that we only need to evaluate the probability $\operatorname{Pr}(N B P(N, c)=d)$ to get the upper bound on blocking probability. To calculate $\operatorname{Pr}(N B P(N, c)=$ $d)$, we shall establish the following theorem.

Theorem 1: For a $\operatorname{VSOB}(N, m, c)$ network, the probability $\operatorname{Pr}(N B P(N, c)=d)$ is given by:

$$
\begin{aligned}
& \operatorname{Pr}(N B P(N, c)=d) \\
& =\sum_{0 \leq d_{1} \leq \min \{(3 / 2) \sqrt{N}, d\}}-\operatorname{Pr}\left(L(N)=d_{1}\right) \cdot \operatorname{Pr}\left(C(N, c)=d_{2}\right) \\
& d_{2}=d-d_{1}
\end{aligned}
$$

Proof: According to the definition of $L(N)$ and $C(N, c)$ in lemma 1. The probability $\operatorname{Pr}(N B P(N, c)=d)=\operatorname{Pr}(L(N)+$ $C(N, c)=d)$ can be separated as the combination of two probabilities $\operatorname{Pr}(L(N))$ and $\operatorname{Pr}(C(N, c))$. Thus, we can easily get formula 2 as shown in theorem 1 .

Theorem 1 shows clearly that the remain problems to get the upper bound on blocking probability are to calculate the probabilities $\operatorname{Pr}\left(L(N)=d_{1}\right)$ and $\operatorname{Pr}\left(C(N, c)=d_{2}\right)$ then we can get the probability of $\operatorname{Pr}(N B P(N, c)=d)$. About the probabilities $\operatorname{Pr}\left(L(N)=d_{1}\right)$ and $\operatorname{Pr}\left(C(N, c)=d_{2}\right)$, given the case there $t_{1}$ connections from input set $I_{\text {even }}-I_{(1 / 2)} \log N$ and $t_{2}$ connections destined for $O_{\text {even }}-O_{(1 / 2) \log N}$ following the $L(N)$, among which there are $k$ connections from $I_{\text {even }}-$ $I_{(1 / 2) \log N}$ and destined for $O_{\text {even }}-O_{(1 / 2) \log N}$, we have the following lemma.

Lemma 2: For a $\operatorname{VSOB}(N, m, c)$ network, the probability 
$\operatorname{Pr}\left(L(N)=d_{1}\right)$ is given by:

$$
\begin{aligned}
& \operatorname{Pr}\left(L(N)=d_{1}\right) \\
& =\sum_{t_{1}=0}^{\min \left\{d_{1}, \frac{\sqrt{N}}{2}-1\right\}} \sum_{t_{2}=0}^{\min \left\{d_{1}, \frac{\sqrt{N}}{2}-1\right\}} \sum_{k=\max \left\{0, t_{1}+t_{2}-d_{1}\right\}} \frac{\operatorname{Pr}\left(t_{1}, k\right) \cdot \operatorname{Pr}\left(t_{2}, k\right)}{\operatorname{Pr}(k)}
\end{aligned}
$$

Where

$$
\begin{aligned}
& \operatorname{Pr}(k)=\left(\begin{array}{c}
\frac{\sqrt{N}}{2}-1 \\
k
\end{array}\right) \cdot \alpha^{k} \cdot(1-\alpha)^{\frac{\sqrt{N}}{2}-1-k} \\
& \operatorname{Pr}\left(t_{1}, k\right)=\sum_{L_{1}+\cdots+L_{\frac{\log N}{2}-1}=k} \sum_{T_{1}+\cdots+T_{\frac{\log N}{2}-1}=t_{1}-k} \\
& 0 \leq L_{i} \leq\left|I_{i}\right|, i=1, \ldots,(\log N / 2)-10 \leq T_{i} \leq\left|I_{i}\right|-L_{i}, i=1, \ldots(\log N / 2)-1 \\
& \left(\prod_{i=1}^{\frac{1}{2} \log N-1}\left(\begin{array}{c}
\left|I_{i}\right| \\
L_{i}
\end{array}\right) \cdot\left(\begin{array}{c}
\left|I_{i}\right|-L_{i} \\
T_{i}
\end{array}\right) \cdot \alpha^{L_{i}} \cdot \beta_{i}^{T_{i}} \cdot\left(1-\alpha-\beta_{i}\right)^{\left|I_{i}\right|-L_{i}-T_{i}}\right)
\end{aligned}
$$

$$
\begin{aligned}
& \operatorname{Pr}\left(t_{2}, k\right)=\sum_{L_{1}+\cdots+L_{\frac{\log N}{2}-1}=k} \sum_{T_{1}+\cdots+T_{\frac{\log N}{2}-1}=t_{2}-k} \\
& 0 \leq L_{i} \leq\left|O_{i}\right|, i=1, \ldots,(\log N / 2)-10 \leq T_{i} \leq\left|O_{i}\right|-L_{i}, i=1, \ldots,(\log N / 2)-1 \\
& \left(\cdot \prod_{i=1}^{\frac{1}{2} \log N-1}\left(\begin{array}{c}
\left|O_{i}\right| \\
L_{i}
\end{array}\right) \cdot\left(\begin{array}{c}
\left|O_{i}\right|-L_{i} \\
T_{i}
\end{array}\right) \cdot \alpha^{L_{i}} \cdot \beta_{i}^{T_{i}} \cdot\left(1-\alpha-\beta_{i}\right)^{\left|O_{i}\right|-L_{i}-T_{i}}\right)
\end{aligned}
$$

Here, $\alpha=r \cdot((\sqrt{N} / 2)-1) /(N-1)$ is the probability that a connection from $I_{\text {even }}-I_{(1 / 2) \log N}$ blocks the tagged path and is destined for $O_{\text {even }}-O_{(1 / 2) \log N}$, and $\beta_{i}$ is the probability that a connection from $I_{i}$ but is not destined for $O_{\text {even }}-O_{(1 / 2) \log N}$, where $r$ is the occupancy probability of an input/output port.

The evaluation of $\operatorname{Pr}\left(C(N, c)=d_{2}\right)$ is summarized in the following lemma.

Lemma 3: For a $\operatorname{VSOB}(N, m, c)$ network, where $\log N$ is even, the probability $\operatorname{Pr}\left(C(N, c)=d_{2}\right)$ is given by the following formula:

$$
\begin{aligned}
& \operatorname{Pr}\left(C(N, c)=d_{2}\right) \\
& =\sum_{s_{1}=0}^{\min \left\{d_{2}, \frac{\sqrt{N}}{2}+\left[\frac{\sqrt{N}}{c+1}\right]\right\} \min \left\{d_{2}, \frac{\sqrt{N}}{2}+\left[\frac{\sqrt{N}}{c+1} \mid\right\}\right.} \sum_{s_{2}=0}^{\sum_{l=\max \left\{0, s_{1}+s_{2}-d_{2}\right\}} \frac{\operatorname{Pr}\left(s_{1}, l\right) \cdot \operatorname{Pr}\left(s_{2}, l\right)}{\operatorname{Pr}(l)}}
\end{aligned}
$$

Where

$$
\begin{aligned}
& \operatorname{Pr}(l)=\left(\begin{array}{c}
\frac{\sqrt{N}}{2} \\
l
\end{array}\right) \cdot \alpha^{l} \cdot(1-\alpha)^{\frac{\sqrt{N}}{2}-l} \\
& \operatorname{Pr}\left(s_{1}, l\right)=\sum_{\frac{L_{\frac{\log N}{2}}}{\frac{\frac{\log N}{2}+1}{2}}=l T_{\frac{\log N}{2}}+T_{\frac{\log N}{2}+1}=s_{1}-l}
\end{aligned}
$$

$$
\begin{aligned}
& \left(\prod_{i=\frac{\log N}{2}}^{\frac{1}{2} \log N+1}\left(\begin{array}{c}
\left|I_{i}\right| \\
L_{i}
\end{array}\right) \cdot\left(\begin{array}{c}
\left|I_{i}\right|-L_{i} \\
T_{i}
\end{array}\right) \cdot \alpha^{L_{i}} \cdot \beta_{i}^{T_{i}} \cdot\left(1-\alpha-\beta_{i}\right)^{\left|I_{i}\right|-L_{i}-T_{i}}\right)
\end{aligned}
$$

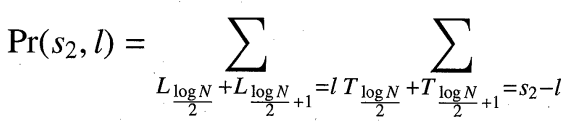

$$
\begin{aligned}
& \left(\prod_{i=\frac{\log N}{2}}^{\frac{1}{2} \log N+1}\left(\begin{array}{c}
\left|O_{i}\right| \\
L_{i}
\end{array}\right) \cdot\left(\begin{array}{c}
\left|O_{i}\right|-L_{i} \\
T_{i}
\end{array}\right) \cdot \alpha^{L_{i}} \cdot \beta_{i}^{T_{i}} \cdot\left(1-\alpha-\beta_{i}\right)^{\left|O_{i}\right|-L_{i}-T_{i}}\right)
\end{aligned}
$$

Here, $\alpha=r \cdot(\sqrt{N} / 2) /(N-1)$ is the probability that a connection from $I_{(1 / 2) \log N} \cup I_{(1 / 2)(\log N+1)}$ passing through the tagged SEs and is destined for $O_{(1 / 2) \log N} \cup O_{(1 / 2)(\log N+1)}$, and $\beta_{i}$ is the probability that a connection from $I_{(1 / 2) \log N} U$ $I_{(1 / 2)(\log N+1)}$ but is not destined for $O_{(1 / 2) \log N} \cup O_{(1 / 2)(\log N+1)}$.

We can prove that the upper bound blocking probability derived above matches the strictly nonblocking condition of a VSOB network [10], as summarized in the following corollary.

Corollary 1: For a $\operatorname{VSOB}(N, m, c)$ network, where $\log N$ is even, the blocking probability $\operatorname{Pr}^{+}$(blocking) given in (1) becomes 0 when $m>(3 / 2) \sqrt{N}+\lfloor\sqrt{N} /(c+1)\rfloor$ if $0<c \leq$ $(1 / 2) \log N$.

3.3 Upper Bound on Blocking Probability When the Number of Stages is Odd

Based on Lemma 1, the blocking probability $\operatorname{Pr}^{+}$(blocking) for a $\operatorname{VSOB}(N, m, c)$ network, where $\log N$ is odd, is given by:

$$
\begin{aligned}
& \left.\operatorname{Pr}^{+} \text {(blocking }\right) \\
& =1-\sum_{d=0}^{\min \left\{\sqrt{2 N}+\left\lfloor{ }^{\sqrt{2 N} /(c+2)], m-1\}} \operatorname{Pr}(N B P(N, c)=d)\right.\right.}
\end{aligned}
$$

The probability $\operatorname{Pr}(N B P(N, c)=d)$ can also be evaluated based on the formula (3)-(10), in which the probability $\operatorname{Pr}\left(L(N)=d_{1}\right)$ is as same as Lemma 2 and the evaluation of $\operatorname{Pr}\left(C(N, c)=d_{2}\right)$ is summarized in the following lemma as Lemma 3.

Lemma 4: For a $\operatorname{VSOB}(N, m, c)$ network, where $\log N$ is odd, the probability $\operatorname{Pr}(N B P(N, c)=d)$ is given by the following formula:

$$
\begin{aligned}
& \operatorname{Pr}(N B P(N, c)=d) \\
& =\sum_{\substack{0 \leq d_{1} \leq \min \{\sqrt{2 N}, d\} \\
d_{2}=d-d_{1}}} \operatorname{Pr}\left(L(N)=d_{1}\right)+\operatorname{Pr}\left(C(N, c)=d_{2}\right) \\
& \quad-\operatorname{Pr}\left(L(N)=d_{1}\right) \cdot \operatorname{Pr}\left(C(N, c)=d_{2}\right)
\end{aligned}
$$

The following corollary indicates that, when $\log N$ is odd, the upper bound blocking probability we derived also matches the condition for a strictly nonblocking VSOB network [10]. 


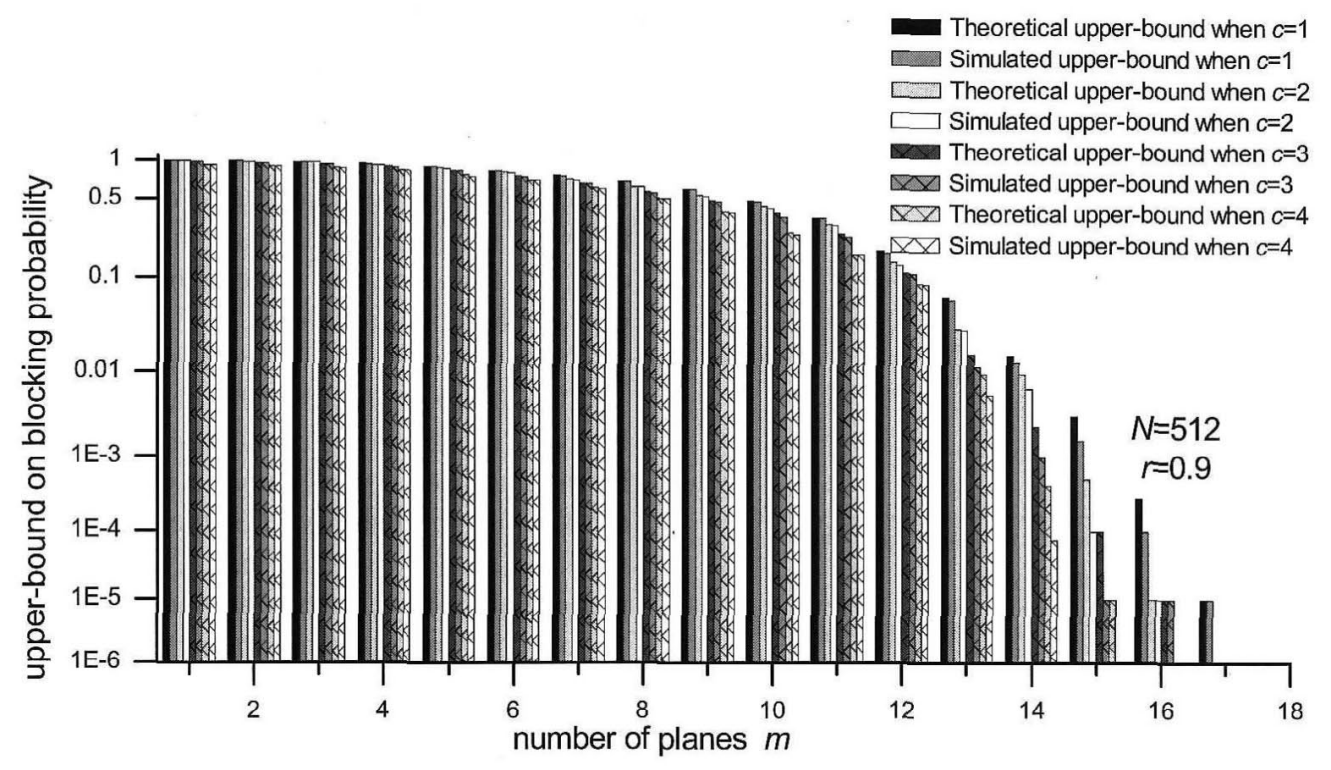

Fig. 3 Blocking probability of $512 \times 512$ VSOB network with $c=1,2,3,4$.

Corollary 2: For a $\operatorname{VSOB}(N, m, c)$ network, where $\log N$ is odd, the blocking probability $\operatorname{Pr}^{+}$(blocking) given in (11) becomes 0 when $m>\sqrt{2 N}+\lfloor\sqrt{2 N} /(c+2)\rfloor$ if $0<c \leq$ $(\log N-1) / 2$.

\section{Experimental Results}

Simulation has been conducted to verify our model on blocking probability (also denoted by $B P$ thereafter) of a VSOB network. Our network simulator consists of the following two modules: the request pattern generator and the request router. The request pattern generator randomly generates a set of connection request patterns for a VSOB network based on the occupancy probability $r$ of an input/output port. To verify the upper bound on $B P$, the "conservative" routing strategy is used in the request router to route the connection requests of a connection pattern through the VSOB network. In the "conservative" routing strategy, we guarantee that all these requests that block a same tagged path will fall within distinct plans. In a $\operatorname{VSOB}(N, m, c)$ network, a plane is blocked if all its tagged paths are blocked. For a connection pattern, if no plane can satisfy the request of the tagged path using a routing strategy, the connection request pattern is recorded as a blocked connection pattern corresponding to the routing strategy. The blocking probability of a routing strategy is then estimated by the ratio of the number of blocked connection patterns to the total number of connection patterns generated. During the simulation, a certain workload is maintained. The workload is measured by the network utilization, which is defined as the probability that an input (output) port is busy.

\subsection{Theoretical Versus Simulated Upper Bounds on $B P$}

In order to verify the derived upper bound from our model, we have examined the $\operatorname{VSOB}(512, m, c)$ network with $c=$ $\{1,2,3,4\}$ for example. For this network configuration, blocking probability is examined by using both the theoretical bound and the simulator for $r=0.9$.

The corresponding results are summarized in Fig. 3. The results in Fig. 3 show clearly that our theoretical model correctly estimates the upper bound on the blocking probability of general VSOB networks under various degree of crosstalk constraint. The results in Fig. 3 also indicate that for a given network configuration, it is possible for us to dramatically reduce the number of planes by tolerating a predictable and negligibly small blocking probability under different crosstalk constraint.

\subsection{Degree of Crosstalk, Blocking Probability and Hard- ware Cost}

Figure 5 illustrates the minimum number of planes estimated by our analytical model for negligible blocking probability under different degree of crosstalk constraint. For comparison, we also show in Fig. 4 the minimum number of planes given by the condition of a strictly nonblocking VSOB network $(c=0)$ implemented by our simulator.

The results in Fig. 5 indicate that, for larger size networks, the hardware costs given by nonblocking condition are considerably higher than that given by the upper bound even for a high requirement of blocking probability. For a switching network with $N=512$, the minimum number of planes given by the nonblocking condition is $47,41,39$, 37,36 for $c=0,1,2,3$ and 4 respectively, while the minimum number of planes given by the implementation are $31,27,23,21,19$ for VSOB with $B P<0.001 \%$, so the $(47-31) / 47 \cong 34 \%,(41-27) / 41 \cong 34 \%,(39-23) / 39 \cong$ $41 \%,(37-21) / 37 \cong 43 \%$ and $(36-19) / 36 \cong 47 \%$ of the hardware can be reduced respectively while a very low 


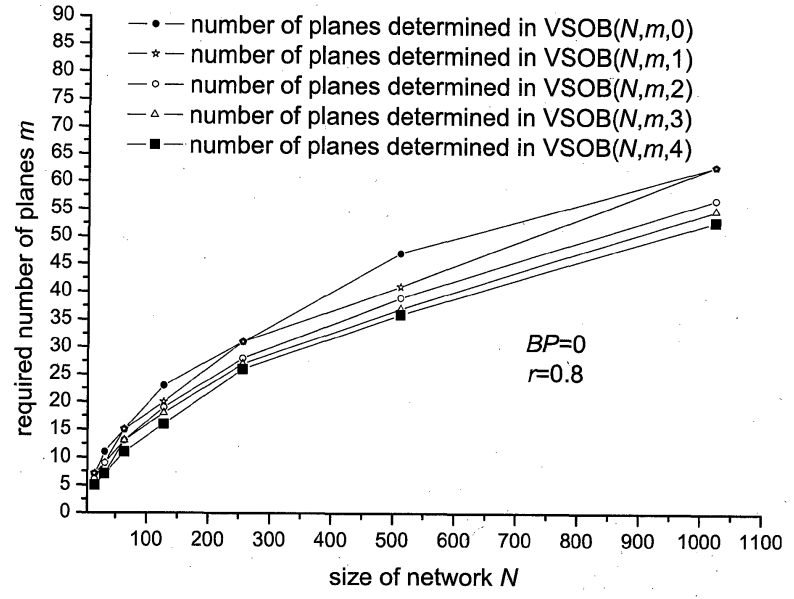

Fig.4 Minimum number of planes for strictly nonblocking VSOB networks.

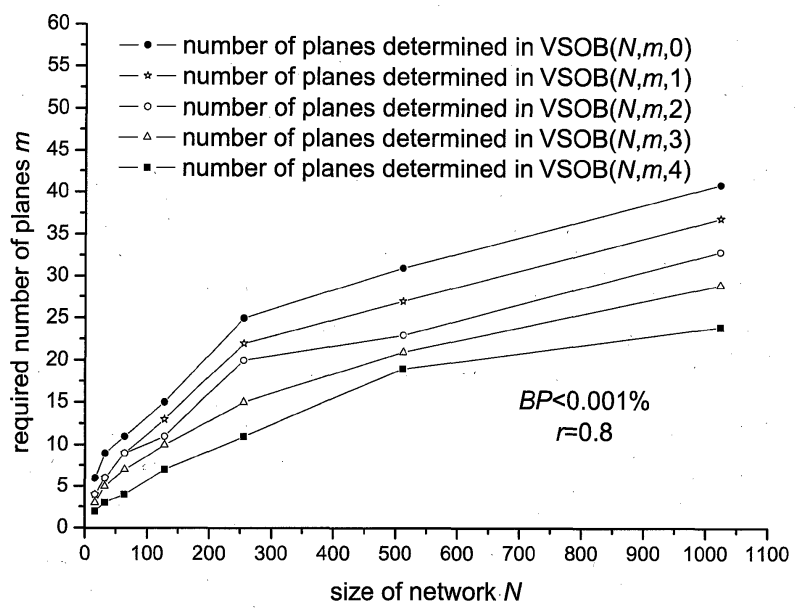

Fig.5 Minimum number of planes for negligible blocking VSOB networks.

blocking probability is guaranteed $(B P<0.001 \%)$. It is interesting to note that by increasing the degree of crosstalk, the hardware cost will be more reduced when the same blocking probability constraint in the VSOB network. It is an efficient tool to find the relationship among hardware cost, blocking probability and degree of crosstalk.

\subsection{Blocking Probability Versus Degree of Crosstalk and Workload}

We have also examined two network configurations, $N=$ 512 and $N=1024$, for upper bound implementation. For each configuration and $r=0.9$, the blocking probabilities were generated under $c=1,2,3,4$. The corresponding results are summarized in Figs. 6 and 7. The comparison results in both Figs. 6 and 7 show clearly that the blocking probability is in sense of crosstalk. And we should note that our upper bound follows closely with the conditions of strictly nonblocking VSOB networks as shown in lemma 1. For example, when $N=512$, the upper-bound blocking probability goes to zero at $m=41$ when $c=1$; goes to zero at $m=39$ when $c=2$; goes to zero at $m=37$ when $c=3$ and goes to zero at $m=36$ when $c=4$. The results in Figs. 6 and 7 also indicate clearly that it is possible for us to dramatically reduce the hardware cost (number of planes) by tolerating a predictable and negligible blocking probability by increasing the degree of crosstalk. So our bound can initiates a graceful tradeoff between hardware cost and overall blocking probability.

The results in Table 1 indicate that, for larger $\operatorname{VSOB}(N, m, c)$ networks, the hardware costs for the nonblocking condition are considerably higher than those given by the proposed upper bound, even under a strict constraint on blocking probability. For the $1024 \times 1024$ VSOB network when $c=1,2,3$ and 4 , the minimum number of planes determined by the nonblocking condition are 63, 57, 55 and 53 , while the minimum number of planes given by our bound are only 39, 36, 32 and 28 for $B P<0.0001 \%$ and $r=1.0$, respectively. The above implies that $(63-$ $39) / 63 \approx 38 \%,(57-36) / 57 \approx 37 \%,(55-32) / 55 \approx 42 \%$ and $(53-28) / 53 \approx 47 \%$ of the hardware cost can be reduced while a very low blocking probability is guaranteed $(B P<0.0001 \%)$. It is also interesting to observe from Table 1 that compared to the variation of $B P$ requirement, the hardware cost estimated by our upper bound is more sensitive to the variation of workload $r$. For the $\operatorname{VSOB}(512, m, c)$ network with $r=1.0$, the minimum number of planes estimated by the upper bound is $17,15,15$ and 14 for the requirement $B P<1 \%$ when $c=1,2,3$ and 4 ; these number increases slightly to $21,19,18$ and 18 when the requirement on $B P$ becomes $B P<0.01 \%$ (100 times stricter), and all the results are much less than the $47,39,37$ and 36 planes required by the nonblocking condition. Again, for the $\operatorname{VSOB}(512, m, c)$ network, we need $20,18,16$ and 15 planes to guarantee $B P<0.01 \%$ when workload is 0.75 , but we require $21,19,18$ and 18 planes to guarantee the same $B P$ requirement when the workload increases to 1.0 (only $25 \%$ higher), and still all these results are much less than the $47,39,37$ and 36 planes required by the nonblocking condition.

\section{Conclusions}

In this paper, we have developed an analytical model for evaluating the upper bound on blocking probability of VSOB networks that under various degree of crosstalk constraint. The derived bound, which agrees with the strictly nonblocking condition of a VSOB network, is proved to accurately depict the overall blocking behavior of the VSOB network by extensive simulation results. The model provides network developers with a guidance of quantitatively determining the impacts of allowing crosstalk and reduction in the number of planes on the overall blocking behavior of a VSOB network in which different routing strategies may be applied. The numerical results of our model can also show how the crosstalk adds a new dimension on the VSOB networks and the effecting of crosstalk on hardware cost and blocking probability. 


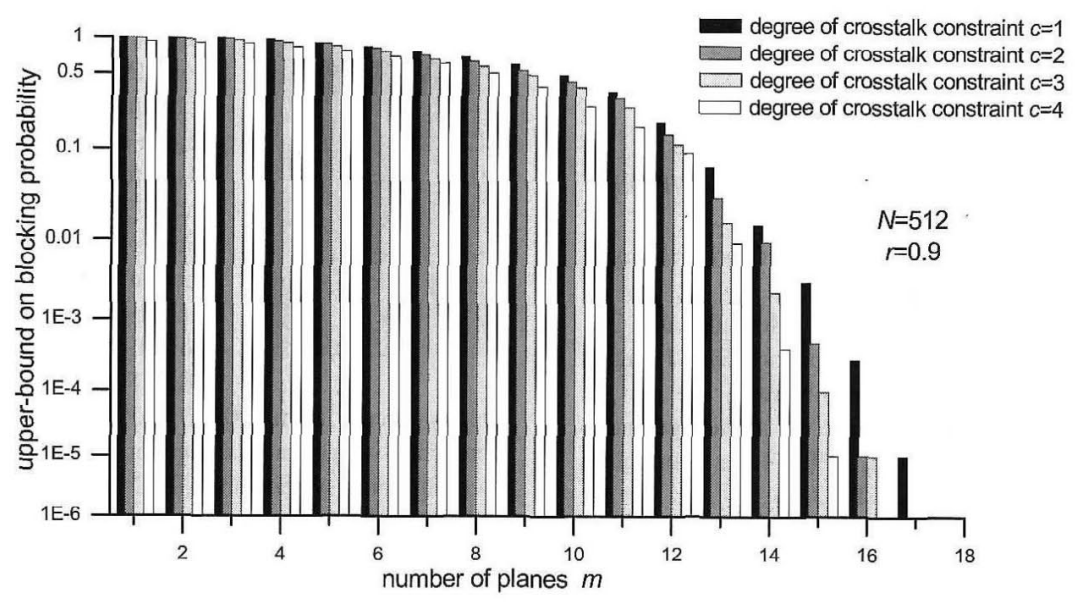

Fig. 6 Blocking probability versus degree of crosstalk in $512 \times 512$ VSOB network.

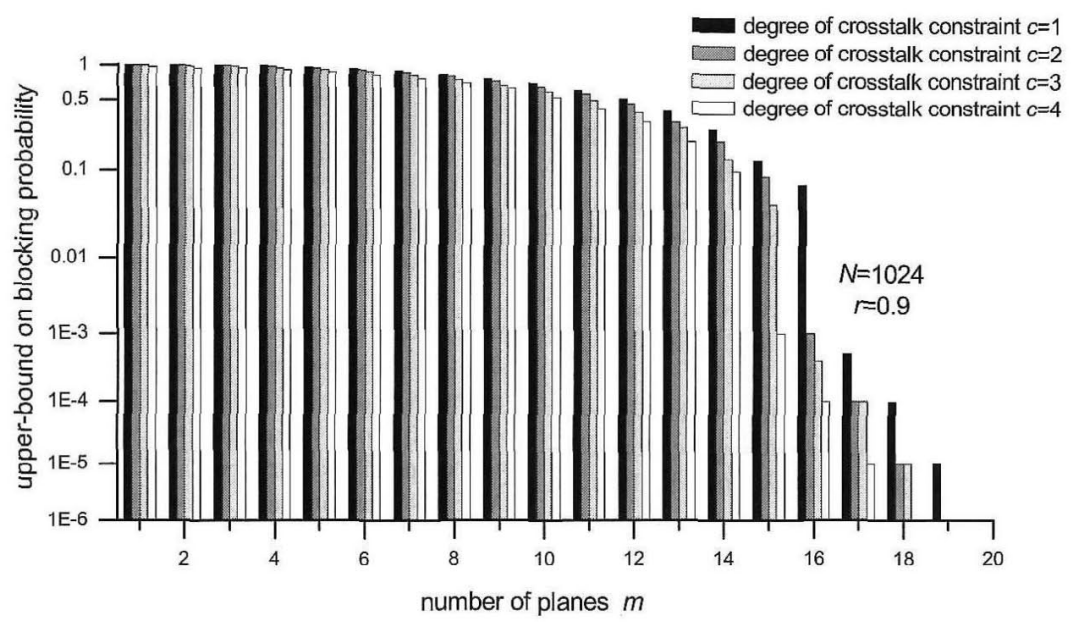

Fig. 7 Blocking probability versus degree of crosstalk in $1024 \times 1024$ VSOB network.

Table 1 Minimum number of planes for $\operatorname{VSOB}(N, m, c)$ networks with different requirements on $B P$ and different workloads.

\begin{tabular}{|c|c|c|c|c|c|c|c|c|c|}
\hline & & \multicolumn{4}{|c|}{$N=512$} & \multicolumn{4}{|c|}{$N=1024$} \\
\hline & & $c=1$ & $c=2$ & $c=3$ & $c=4$ & $c=1$ & $c=2$ & $c=3$ & $c=4$ \\
\hline$B P=0$ & $r=1.0$ & 41 & 39 & 37 & 36 & 63 & 57 & 55 & 53 \\
\hline \multirow{3}{*}{$B P<0.0001 \%$} & $r=0.5$ & 21 & 19 & 16 & 13 & 30 & 29 & 26 & 24 \\
\hline & $r=0.75$ & 28 & 23 & 21 & 19 & 37 & 33 & 29 & 24 \\
\hline & $r=1.0$ & 33 & 26 & 22 & 21 & 39 & 36 & 32 & 28 \\
\hline \multirow{3}{*}{$B P<0.01 \%$} & $r=0.5$ & 17 & 14 & 12 & 11 & 22 & 20 & 19 & 17 \\
\hline & $r=0.75$ & 20 & 18 & 16 & 15 & 26 & 22 & 21 & 19 \\
\hline & $r=1.0$ & 21 & 19 & 18 & 18 & 29 & 27 & 25 & 24 \\
\hline \multirow{3}{*}{$B P<1 \%$} & $r=0.5$ & 13 & 12 & 11 & 10 & 15 & 13 & 12 & 12 \\
\hline & $r=0.75$ & 15 & 13 & 12 & 10 & 18 & 17 & 17 & 16 \\
\hline & $r=1.0$ & 17 & 15 & 15 & 14 & 20 & 18 & 18 & 16 \\
\hline
\end{tabular}

This analytical model can further help network designers to find the optimal VSOB structure for building an optical switching network with a specified constraint on blocking probability. An important conclusion drawn from our work is that the hardware cost of a VSOB network can be reduced dramatically while a negligible small blocking probability and reality crosstalk are guaranteed. We expect that modeling method employed in this paper will help deriving the upper bound on blocking probabilities of other types of optical switching networks.

\section{Acknowledgments}

This research is partly supported by the Grand-In-Aid of science research from Japan Society for the Promotion of Science and Strategic International Cooperative Program, 
Japan Science and Technology Agency (JST).

\section{References}

[1] H.S. Hinton, An Introduction to photonic Switching Fabrics, Plenum, New York, 1993.

[2] R. Ramaswami and K.N. Sivarajan, Optical Networks, Morgan Kaufmann Publishers, ISBN: 1-55860-655-6, 2002.

[3] V.R. Chinni, T.C. Huang, P.-K.A. Wai, C.R. Menyuk, and G.J. Simonis, "Crosstalk in a lossy directional coupler switch," J. Lightwave Technol., vol.13, no.7, pp.1530-1535, July 1995.

[4] C. Kruskal and M. Snir, "The performance of multistage interconnection networks for multiprocessors," IEEE Trans. Commun., vol.COM-32, no.12, pp.1091-1098, Dec. 1983.

[5] F.T. Leighton, Introduction to Parallel Algorithms and Architectures: Arrays, Trees, Hypercubes, Morgan Kaufmann, 1992.

[6] G.R. Goke and G.J. Lipovski, "Banyan networks for partitioning multiprocessor systems," Proc. 1st Annu. Symp. Comp. Arch., pp.21-28, 1973.

[7] J. Patel, "Performance of processor-memory interconnections for multiprocessors," IEEE Trans. Comput., vol.C-30, no.10, pp.771780, Oct. 1981.

[8] G. Maier and A. Pattavina, "Design of photonic rearrangeable networks with zero first-order switching-element-crosstalk," IEEE Trans. Commun., vol.49, no.7, pp.1268-1279, July 2001.

[9] M.M. Vaez and C.T. Lea, "Wide-sense nonblocking banyan-type switching systems based on directional couplers," IEEE J. Sel. Areas Commun., vol.16, no.7, pp.1327-1332, Sept. 1998.

[10] M.M. Vaez and C.T. Lea, "Strictly nonblocking directional-couplerbased switching networks under crosstalk constraint," IEEE Trans. Commun., vol.48, no.2, pp.316-323, Heb. 2000.

[11] C. Yu, X. Jiang, S. Horiguchi, and M. Guo, "Overall blocking behavior analysis of general banyan-based optical switching networks," IEEE Trans. Parallel Distrib. Syst., vol.17, no.9, pp.1037-1047, Sept. 2006

[12] X. Jiang, H. Shen, M. Khandker, and S. Horiguchi, "Blocking behaviors of crosstalk-free optical banyan networks on vertical stacking," IEEE/ACM Trans. Netw., vol.11, no.6, pp.982-993, Dec. 2003.

[13] X. Jiang, H. Shen, and S. Horiguchi, "Blocking probability of vertically stacked optical banyan networks under random routing," Proc. GLOBECOM 2003, San Francisco, USA, Dec. 2003.

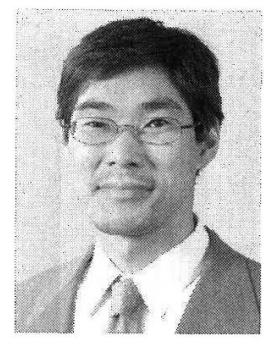

Yasushi Inoguchi received B.E. degree from Department of Mechanical Engineering, Tohoku University in 1991, and received M.S. degree and Ph.D. from JAIST (Japan Advanced Institute of Science and Technology) in 1994 and 1997, respectively. He is currently an Associate Professor of Center for Information Science at JAIST. He was a research fellow of the Japan Society for the Promotion of Science from 1994 to 1997. He was also a researcher of PRESTO program of Japan Science and Technology Agency from 2002 to 2006. His research interest has been mainly concerned with parallel computer architecture, interconnection networks, reconfigurable system, and GRID architecture. Dr. Inoguchi is a members of IEEE and IPS of Japan.

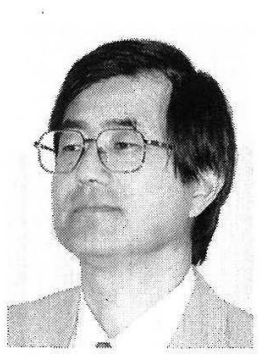

Susumu Horiguchi received the B.Eng., M.Eng. and Ph.D. degrees from Tohoku University in 1976, 1978 and 1981 respectively. $\mathrm{He}$ is currently a professor and Chair of Department of Computer Science, the Graduate School of Information Science, Chair of Department of Information Engineering, Faculty of Engineering, Tohoku University. He was a visiting scientist at the IBM Thomas J. Watson Research Center from 1986 to 1987 . He was also a professor in the Graduate School of Information Science, JAIST (Japan Advanced Institute of Science and Technology). He has been involved in organizing international workshops, symposia and conferences sponsored by the IEEE, IEICE, IASTED and IPS. He has published over 150 papers technical papers on optical networks, interconnection networks, parallel algorithms, high performance computer architectures and VLSI/WSI architectures. Prof. Horiguchi is a senior member of IEEE and member of IPS and IASTED.

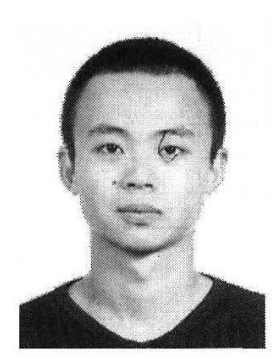

Chen Yu received the B.Sc. and M.Eng. degrees in 1998 and 2002, from Wuhan University, Wuhan, China, and received Ph.D. degree in 2005 from Tohoku University, Japan. He is now with JAIST (Japan Advanced Institute of Science and Technology) as a JSPS (Japan Society for the Promotion of Science) Postdoctoral Fellow. He was a JST postdoctoral researcher for JAIST from Oct. 2005 to Mar. 2006. He was the research assistant and teaching assistant in the Graduate School of Information Science, Tohoku University, from Apr. 2004 to Sep. 2005, and researcher of JAIST from 2002 to 2004, respectively. His research interests include interconnection networks, optical switch networks, WDM networks, Grid computing, network coding and data mining. 